\title{
30 \\ REASSEMBLING THE INFORMATION TECHNOLOGY INNOVATION PROCESS: An Actor Network Theory Method for Managing the Initiation, Production, and Diffusion of Innovations
}

\author{
Gerardo Zendejas \\ Mike Chiasson \\ University of Lancaster \\ Lancaster, UK
}

\begin{abstract}
This paper will propose and explore a method to enhance focal actors' abilities to enroll and control the many social and technical components interacting during the initiation, production, and diffusion of innovations. The reassembling and stabilizing of such components is the challenging goal of the focal actors involved in these processes. To address this possibility, a healthcare project involving the initiation, production, and diffusion of an IT-based innovation will be influenced by the researcher, using concepts from actor network theory (ANT), within an action research methodology (ARM). The experiences using this method, and the nature of enrolment and translation during its use, will highlight if and how ANT can provide a problem-solving method to help assemble the social and technical actants involved in the diffusion of an innovation. Finally, the paper will discuss the challenges and benefits of implementing such methods to attain widespread diffusion.
\end{abstract}

Keywords Innovation, diffusion, enrolment, translation

Please use the following format when citing this chapter:

Zendejas, G., and Chiasson, M., 2008, in IFIP International Federation for Information Processing, Volume 287, Open IT-Based Innovation: Moving Towards Cooperative IT Transfer and Knowledge Diffusion, eds. León, G., Bernardos, A., Casar, J., Kautz, K., and DeGross, J. (Boston: Springer), pp. 527-539. 


\section{THEORETICAL FOUNDATIONS}

\subsection{Innovation Diffusion Theory (IDT)}

Roger's definitions of innovation and diffusion are by now generally accepted as the starting point for most IDT research. Innovation is understood as an idea, practice, or object that is perceived as new by an individual or other unit of adoption. Diffusion is defined as the process by which an innovation is communicated through certain channels over time among the members of a social system (Rogers 1962).

Although Roger's IDT has vastly influenced subsequent diffusion research, one of its main limitations in its practical and research applications is an assumption that innovations are largely unchanged during the diffusion process, thus restricting its relevance to many aspects of information systems and information technology in which the innovations continue to change and adapt during diffusion.

Slappendel (1996) performed a comprehensive literature review of research on innovations in organizations and provided a framework to classify the existing research work in three categories based on innovation causality: the individualist, the structuralist, and the interactive process perspectives.

The individualist perspective assumes that individuals are the main source of change in organizations. In contrast, the structuralist perspective assumes that existing organizational characteristics enable innovation. Both perspectives consider innovations as relatively static objects and the innovation process as a linear continuum of design, development, and adoption phases.

The interactive perspective assumes that innovation is a dynamic and continuous phenomenon of change over time in which various factors dialectically influence each other. Since the actions of innovative individuals cannot be divorced from the activities of other individuals or from the organizational structures within which they operate, innovation is the result of the continuous interaction of the actions of individuals, structural influences, and the innovation itself (Kautz and Nielsen 2004).

On the other hand, the increasing decentralization of IT innovation processes, characterized by the rapid formation and dissolution of innovation networks composed of heterogeneous and complex actors must be taken into account. To do so, we believe that a method, based on actor network theory (ANT), is required. The interactive perspective provides the best starting point for the analysis. We will show that a sociotechnical theoretical approach is needed to reassemble the IT innovation process and to facilitate a problem-solving method for managing it.

\subsection{Actor Network Theory (ANT)}

There are many definitions of ANT, but probably the best one is also the shortest: ANT is the science of associations. It provides an epistemological basis upon which human and nonhuman actors can be enrolled and associated within networks, engaging in collective action by translating the various actors' interests into a common force that will help transform claims into facts or innovations (Latour 1999). So the context, using 
ANT, is the set of human and nonhuman actors fused together into networks, mobilized actors engaged in collective action to realize collective and individual interests (Latou 2005).

Therefore, we believe that ANT can be instrumental in selecting, understanding, and influencing the collective of social and technical factors interacting during the innovation process. First of all, ANT would be effective in recasting the IT innovation context as a dynamic, distributed interplay of human and nonhuman actors pursuing the establishment of irreversible and maintainable innovations by enrolling providers and receivers, and by searching these various translations in establishing agreements for moving collectively through the different phases of the innovation process: initiation, production, and diffusion.

Secondly, if considered as a method for facilitating enrolment and translation, an ANT approach could be viewed as a distributed project management method, focused on the generation and stabilization of networks. To develop and assess a particular method informed by ANT, we would need to follow and observe the language and actions of actors during all phases of the innovation process. Such a method would be consistent with ANT's ontology, which is primarily based on the fact that reality is a linguistic and political outcome of a stabilized and negotiated interplay among actors. ANT approaches to reflection and action would focus on understanding the network stabilization process and its irreversibility, that is, the specific interplay among actors in a network that results in "black boxes" (Cordella and Shailch 2006).

A research and problem-solving approach based on this, however, would differ from traditional "opening the black-box" studies of ANT, which primarily focus on the retrospective processes that made a network stable, and would turn to the "tracks the process before the blackbox gets closed" (Lanzara 1999). This would move beyond the traditional use of ANT in IS as an interpretative lens. For tracking the stabilization process, an active and longitudinal research approach is required.

Further to ANT's theoretical, epistemological, and ontological insight in reassembling the IT innovation process, ANT can also inform the nature and processes involved in the uptake of innovations. ANT theorists would thus consider traditional approaches to IDT as incomplete at best, flawed at worst, in their implicit assumptions that society is the medium through which innovations diffuse more successfully or not depending upon the level of resistance of particular groups. These groups might eventually change their mind one way or the other in supporting or not the innovation based primarily on their psychological profiling. These assumptions suggest that the diffusion of innovations arises in a society that is initially disengaged and ignorant of an innovation, but through heroic innovators, is eventually able to overcome degrees of social resistance in adopting innovations.

In contrast, an ANT view on innovation uptake, from translation, would claim that uptake dynamics are linguistic, political, and social, involving the convincing heterogeneous actors by means of translating their interests through bridging dialogues, which tenuously enrol them into a socio-technical ensemble. The implications of such a view on innovation uptake suggest that diffusers have a different and initially more difficult task of reassembling and stabilizing a socio-technical set of relations across technology and participants, which often span long times and large distances. This paper will explore an ANT-based notion of innovation uptake. 


\subsection{Action Research Methodology (ARM)}

In accord with ANT's ontology, the goal of the researcher is to understand the associations that stabilize the network and make the innovation maintainable and irreversible, implying that the researcher's role can include an active part in the production of networks, participating and observing how actors create reality. Therefore, an action research methodology (ARM) could use ANT's ontology to lay the groundwork for both documenting and participating in the construction of a collective reality.

Furthermore, Chiasson et al. (forthcoming) suggest that action research lends itself to pluralist approaches that facilitate the production of both problem-solving and theoretical knowledge in an iterative and cyclical process.

\subsection{Innovation Management Techniques (IMTs)}

Since this paper is arguing that an ANT-based method is required to reassemble and stabilize the IT distributed innovation network and ultimately promote widespread diffusion of innovations, it is relevant to study the application and effects of innovation management techniques (IMTs) used in the production and diffusion of innovations. Theoretical and practical outcomes from these studies are likely to contribute to the construction of such methods.

Terré i Ohme (2002) conducted a comprehensive review of existing IMTs within the European Community. His examination indicated that despite the fact that numerous IMTs have been developed and made available to European organizations involved with innovation, its diffusion in time and space is rather limited. He developed a comprehensive guide for managing innovation based on Chiesa et al. (1996) and Brown (1997). The guide was aimed at small and medium-sized enterprises to help them assess their capacity for innovation. The guide facilitates the process for identifying the basic issues at the organization level for improving its capacity for innovation, and it is currently used as an audit and consulting tool. We believe that such a tool will also assist the process of identifying social and technical components affecting innovation and the construction of the ANT-based method.

Hidalgo and Albors (2008) created a comprehensive study of the many social and technological components involved in the development and use of methods to manage innovation in a knowledge-driven economy. Their review identifies the main IMTs currently in use for improving competitiveness by means of implementing a knowledge management approach. Therefore, it concentrates on those IMTs that focus on knowledge as a relevant component of the innovation process. The review concluded that a knowledge-driven economy affects the innovation process and approach and, therefore, traditional IDT is challenged. A more comprehensive review of Hidalgo and Albors' work is pending for determining potential contributions based on knowledge-driven IMTs for constructing the required ANT-based method.

On the other hand, Hidalgo and Albors' work promotes awareness with regard to recent developing trends of methodologies and tools for supporting business innovation management. Both public and private sector organizations are involved in this effort. However, the proliferation of such methodologies together with the lack of national and 
international guidelines and standards would likely create an important challenge for standardization and uptake of such methodologies and tools in the long term. Therefore, the efforts of initiating, producing, and diffusing the required ANT-based method will carefully follow ANT's prescription for creating the proper socio-technical ensemble for the widespread uptake of innovations.

\section{RESEARCH PLAN}

\subsection{Research Design}

The data for producing and assessing the ANT-based method will flow from a healthcare project involving the initiation, production, and diffusion of an Electronic Synoptic Report (ESR) system across the entire province of Alberta, Canada, with an initial target audience of 250 health providers dispersed across seven health regions. ESR is an innovation because it would replace the traditional procedural dictated report, which has been used for documenting scope-driven procedures ever since the scopes were introduced into medical diagnostic practices. Manually produced dictated reports are today the status quo for most reporting purposes within the medical industry, despite numerous but rather uncoordinated efforts in diffusing automatic forms of reporting.

The ESR project will be influenced and documented using an IMT based on ANT's epistemology and ontology. The project manager will also act as a researcher and spokesperson for ESR, employing an ARM. The interventions will focus on the various arguments and reactions of actors to the discussions of various spokespersons about the IT innovation process. Theoretically, such interventions might then serve as the basis for designing a general problem-solving method to facilitate enrolment and translation in the initiation, production, and diffusion of innovations. Following Chiasson et al.'s (forthcoming) views on ARM, the successful implementation of such a problem-solving method will explore the applicability of ANT's theoretical knowledge to a practical problem setting, and in the process, contribute to ANT and IT-based versions of ANT.

\subsection{Research Methodology}

To explore how the ESR project is mobilized using ANT as an approach, the researcher will facilitate, observe, and document the early stages of the initiation phase that will consist of a handful of expert panel gatherings aimed at reaching consensus as to what the innovation shall be, which organizational functions shall have the required IT solution, and which vendor might be best suited to facilitate the solution. During these gatherings, an empirical grasp of which techniques promote (or not) the enrolment and translation of heterogeneous groups' interests will be gained and used for designing a preliminary problem-solving method.

The researcher will be involved in the production phase, using ANT both as a theoretical perspective and as a problem-solving method, for promoting enrolment of healthcare providers to the ESR's network and facilitating the required translations to 
convert the individual interests into a collective force. During this time, the researcher will employ semi-structured interviews to capture arguments and reactions of actors to the ANT-influenced production phase discussion. The data will be analyzed and the outcomes (both planned and unplanned) will be used to evaluate and explore various problem-solving methods. During this phase we expect to identify, select, understand, and influence the collective of social and technical factors that later will be used for designing the required ANT-based method and reassembling the IT innovation processes.

Finally, the outcomes of the diffusion phase will be measured and analyzed in terms of the improvements gained by implementing the ANT-based method in this project as it compares with a parallel project not benefiting from it. The "control" project has been identified and will also comprise the initiation, production, and diffusion of another ESR technology. The ESR project team has been isolated from the control project team to ensure valid measurement and comparison of variables. The timing of these projects is very similar, at least from the planning perspective. Their budgets are also comparable. Based on the projects' similarities and the independence between projects, we expect to obtain reliable data in terms of diffusion improvements or setbacks.

\subsection{Preliminary Research Outcomes of the Initiation Phase}

Due to the fact that the initiation phase of this project started roughly a year before the research and intervention activities, a retrospective collection of events will be used to understand early enrolment and translation practices for this project. As no researchlead influence was exerted at the time of the initial events, the outcomes of this analysis might be used to compare and contrast the ones obtained before and after the researcher interventions were introduced.

The innovation initiation phase of the Electronic Synoptic Report (ESR) project started on 2006 and it consisted of individual efforts from the Alberta Colorectal Cancer Screening Program (ACRCSP) management team members to enrol others into the idea of using synoptic reporting to facilitate standardized collection of data on colonoscopy procedures. Its purpose was to implement an ongoing quality improvement process of colonoscopy practices at a provincial level.

Synoptic reporting is a synopsis or summary of data that uses standardized nomenclature and data as well as a consistent report structure. Colonoscopy is a procedure in which a long flexible viewing tube known as the colonoscope is threaded up through the rectum for the purpose of inspecting the entire colon and rectum. If there is an abnormality, the colonoscope allows for taking a biopsy of it and/or removing it.

By April of 2007, the ACRCSP published the first hard proof of the innovation's existence, a document titled "Enhanced Delivery of Colorectal Cancer Screening through Information Management: The Development of an Automated Synoptic Colonoscopy Reporting System." This document was distributed among many potential actors and their reactions generated an initial set of controversies to be settled.

The content of the document denoted a moderate degree of technical determinism; the technological role on enhancing the delivery of colorectal cancer screening was overemphasized, with most of the social components involved in the IT innovation process ignored. It is noted that many of the missing social components could be brought to light by means of including a change management methodology. 
A primitive network composed of a handful of leading colonoscopy practitioners and the ACRCSP medical lead for the colorectal program emerged in the form of an expert panel. The panel's initial work on settling the above mentioned controversies was primarily an evolutionary decision-making process characterized by the debate arising from opposing views in terms of personal versus regional and regional versus provincial goals. A second set of controversies arose from the first set, providing a list of contrasting views regarding the required functionality and the potential ways to supply it.

The ACRCSP assumed the lead role in facilitating, documenting, and analyzing the outcomes of this entire phase, which was mainly composed of three face-to-face expert panel meetings, plus a handful of video and phone conferences occurring within a year's time. It is the intention of the researcher to strengthen the lead role assumed by the ACRCSP up to the extent of transforming it, along with its innovation artefact, into an obligatory passage point (OPP), using ANT terms; the researcher's goal is that anyone in the province needing to implement a synoptic reporting artefact would consult with the ACRCSP experts. Next, the outcomes from these expert panel meetings were analyzed and used to produce an initial set of problem-solving scenarios for managing the initiation phase.

\subsubsection{First Expert Panel Meeting (August 21, 2007)}

The conversation focused on understanding discrepancies in the production and distribution of colonoscopy related data and reports across the province. Many of these differences were related to personal preferences, regional constraints, and lack of provincial guidelines on the matter. These differences were perceived as a barrier to establishing a provincial quality improvement process. Empirically, then, future enrolment techniques would have to consider the influence of personal preferences, regional constraints, and the upcoming availability of provincial guidelines.

Actors made important progress in defining the data elements required regionally to establish an effective ESR project. One of the outcomes of this stage - a standardized data set - would later indicate the existence of a regional versus provincial controversy, mainly characterized by the different uses and amount of data required at the regional versus the provincial level. While the regions were interested in collecting large amounts of procedural and clinical data, the province was learning that the actual data requirements to establish an ongoing quality improvement process were rather limited, consisting of approximately 20 data fields.

Flowing from the data elements conversation, an empirical grasp as to which techniques promoted enrolment at the regional level was gained. Such techniques would have to provide means for translating numerous well established interests such as clinical sufficiency of the data, adequacy of the resulting procedural report for legal and record purposes, restricted regional report accessibility, and the ability of managing digital images. It is expected that the ANT-based problem-solving method would include careful displacements of regional interests by avoiding or dissolving geographical boundaries.

Translating the above mentioned regional interests into the provincial ones of the ACRCSP was achieved by its medical lead by implementing the following strategy: "The success of a province wide program is largely a by-product of supporting the needs of regional programs first." Such a strategy matches very closely Latour's (1987) first 
translation strategy, consisting of catering to others' interest. Due to the known limitations of the first strategy, the researcher will focus on applying the appropriate tactics to understand and enrol the interests and goals of actors, aiming to achieve a translation strategy comparable to Latour's fifth strategy: becoming indispensable.

Reviewing the ACRCSP's managerial context is relevant in terms of understanding currently established practices and its effect on the ESR project. The ACRCSP does not follow a project management methodology (PMM) for implementing its projects, however this situation is changing as a result of increased project complexity and the need to manage multiple projects in parallel. It is believed that the lack of a PMM has also had a positive influence on developing actors' negotiation skills above and beyond what is traditionally offered by PMM. As a result, holding all other things equal, it is suspected that implementing effective translation strategies may be easier than with well established practitioners of PMM or similar methodologies.

Therefore, the incipient status of PMM at the ACRCSP might positively influence the implementation of the ANT-based method as the implementer would face significantly less resistance than otherwise. By the same token, implementing supplementary ITMs might be easier too. It must be noted that ITMs might benefit organizations differently and so selecting the right ITMs becomes a highly contextual exercise. The virtual lack of PMM is an unexpected situation that might serve further research purposes in terms of comparing the effects of ANT's interventions in organizations with and without PMMs.

\subsubsection{Second Expert Panel Meeting (October 15, 2007)}

For the most part, the conversation gravitated around the different ways in which the ESR project could be implemented, considering existing off-the-shell solutions and one in-house option. This was likely the case due to the sufficient level of comfort achieved by actors with regard to the first controversy's settlement, facilitated by the translation strategy outlined during the first meeting. Implementation options and their related uptake factors were introduced into the conversation, indicating actor's willingness to move forward into the production phase of the project.

The language of the actors at this stage indicated that the once primitive network was evolving into a stronger one, searching for preliminary ways to achieve stabilization, with all the regional and provincial goals clarified, a translation to amalgamate them, and, for the most part, personal goals properly translated into network ones. Their approach is mostly consistent with the four-stage model of group development produced by Tuckman (1965). The model identifies the forming, storming, norming, and performing stages. The fact that the network was seeking stabilization can be interpreted as a motion to depart from storming into norming.

Comparing stabilization with norming is relevant due to the fact that the network, at that point in time, had not been directly influenced by the researcher using ANT concepts, and the only known approach to the group for measuring the network/team success was Tuckman's model. Other indications of network development at the time included the establishment of clear leadership, and the willingness to expand the network with more colonoscopy experts, IT experts, healthcare consultants and a "nucleus group," as defined by the ESR network chair, for starting the production phase of the project by 
producing the first synoptic report prototype. Given this level of network maturity, we will call it the ESR network from this point forward.

As per the technology conversation, the ESR network created three options for producing the ESR artefact: bare bones, add on, and complete package. These tags strongly related to scope extent; however, their descriptions were based on the advantages or disadvantages that each would likely produce if chosen. These descriptions were very comparable in form and content with traditional SWOT analysis, with the exception that the actors merged strengths and opportunities under the advantage section, weaknesses and threats under the disadvantages section.

The innovation uptake factors were also included as part of the advantages and disadvantages. Assumptions were made in terms of which option might produce a particular uptake level; however, they were not based on any particular uptake forecast model or theory. Uptake expectations mainly rely on previous experiences and observations from actors and, therefore, they are quite possibly out of context. More concerning, the assumptions used by the ESR network only partially considered the fact that diffusing an innovation is fundamentally different than diffusing a well known and already accepted technology.

During the ANT intervention time, the researcher will develop different activities to promote the understanding and application of ANT's views on technology uptake. Contrasting and comparing ANT's views with traditional IDT views will be done to aid the learning process due to the fact that most uptake assumptions recorded during this meeting were somehow related to Roger's views on uptake.

One of the most challenging tasks during this learning process will consist of raising awareness within the ESR network about the dangers of oversimplifying the effect of uptake strategies as it is often during this last phase when most projects fall apart. Furthermore, convincing the actors with regard to the positive implications of adopting ANT's uptake model, including the claim that it would avoid and productively work with the resistance of particular groups during the diffusion phase, will enhance the overall acceptance of problem-solving methods based on ANT as a preferred approach for dealing with IT innovation projects.

\subsubsection{ANT Intervention Begins (February 11, 2008)}

As a result of the ESR network's willingness to expand, the next logical step for the network consisted of creating and staffing the ESR production team by hiring a program manager, a project coordinator, and a project manager. Up until this point in time, the medical lead acted as project manager. The transition was characterized by a soft learning curve and some essential interaction with the other network members. The project manager (the lead author) was initially asked to produce a comprehensive set of project management documents and a technology assessment among three different IT vendors, and to reach consensus among network members.

The first ANT intervention consisted of supporting a learning process to improve the ESR network awareness of the many social and technical components that support the initiation, production, and diffusion of innovations. Previous to this intervention, a great majority of the actors considered stakeholders, both internal and external, as the primary force resisting or preventing the free flow of innovations. This is mainly consistent with 
traditional IDT views on innovation uptake. As it is the purpose of this paper to support ANT's views on uptake, the researcher started to spread the notion of enrolment and translation, as well as the implications of using the translation theory instead of the diffusion theory for explaining uptake dynamics.

The researcher used an oversimplification to convey the concepts of both enrolment and translation; by enrolling all internal and external stakeholders since the initiation phase and during the entire production phase of the project, minimal or not resisting force would act against the collective efforts in achieving wide diffusion, since the enrolled stakeholders will support the process. Enrolling stakeholders can be done by translating their explicit interest into the ones pursued by the innovator. The negotiation efforts will include subtle and incremental displacements of explicit personal or subgroup interests into the larger ones of the collective.

The results of this dialect and oversimplified version of ANT are showing some promising initial results. Later conversations with actors denoted an increased awareness of technical and social components affecting the innovation process. Furthermore, the network notion of collaboration evolved a step or two as the network is now considering not only its obvious short term benefits - improved quality and efficiency — but also the possibility of working with what were considered the resisting forces of dissenters during the diffusion phase.

\subsubsection{Third Expert Panel Meeting (April 25, 2008)}

The language of the actors at this stage clearly indicated the preliminary effects of the ANT interventions. However, these effects were showed unevenly among the ESR network members as a result of the recent network expansion and the challenges of propagating the influence across geographically disperse actors. This is perhaps the most important challenge identified by the researcher at this point in time: exerting ANT influences beyond geographical borders.

The researcher called this meeting to facilitate a decision-making process with regard to selecting an IT vendor as the basis for building the ESR artefact. After due deliberation, the ESR network's chairman came to the conclusion that the ESR network didn't have enough knowledge of all social and technical components affecting innovation practices on every single regional health authority (RHA) in Alberta and, therefore, it was not prepared to produce a top-down policy to enforce adoption of a particular solution. Instead, it was prepared to influence the decision process on every region by chairing informational gatherings, producing environmental assessments, and elaborating a regional recommendation once all social and technical components have been identified, analyzed, and ranked according to the regional priorities and strategies.

The effects of such a decision are expected to be very relevant for the matters of expanding the network first, which now include a critical mass of actors to move forward the innovation artefact, and by further positioning the ACRCSP as the OPP, making sure that essential components of the overall program would be included during the initiation phase of each region, and that those components could be made operational at the provincial level at a later time.

Indeed, at this point in time the ESR network was in need of including many more actors. For example, selecting at least two pilot regions to implement the yet-to-be- 
produced ESR innovation was required. It is prudent by now to characterize and differentiate a number of new potential actors. Alberta is broken into nine health regions, two of which are significantly larger than the others as they are both located at the two main metropolitan areas of the province. Both the Calgary Health Region and Capital Health Region in Edmonton account for almost 80 percent of the patients in the province.

As a logical rule of thumb, the larger the region, the more stakeholders and the more complex it is. Both metropolitan regions already moved unilaterally toward creating their own ESR artefact. In fact, the Calgary Health Region has already completed both the initiation and production phases of their project. The diffusion phase is on the go and it achieved some success thanks to their centralization strategy, which concentrates the delivery of colonoscopy services in a single location that is fully equipped with the ESR artefact and staffed by mostly ESR supporters.

The ACRCSP expectations on the pilot sites mainly consist of gaining experience over the complete implementation process of the ESR artefact. Therefore, neither metropolitan area is adequate for fulfilling this purpose. On the other hand, the expert panel was poorly represented in terms of all other regions, having originally only one representative from the non-metropolitan regions, the Chinook region. An unexpected representative from the Peace Country region showed up the meeting. As part of the efforts toward stabilizing the network, the researcher will focus on expanding the ESR network with representatives of all regions.

It must be noted that it was not possible to exert significant ANT influence during this third meeting, as the time was mainly used by presenters from the potential IT technology providers. The closing remarks emphasized as much as possible the importance of working in collaboration with the ACRCSP and, therefore, promoted the mobilization of turning the ACRCSP and its innovation artefact into an OPP, however, significantly more work is required in this direction. The ACRCSP team remains optimistic with regard to securing at least the two pilot projects before the end of 2008 .

\section{EXPECTED RESULTS AND CONTRIBUTIONS}

\subsection{Expected Results}

The successful implementation of ANT-based problem-solving methods will determine the applicability of our specific implementation of ANT concepts as a practical problem-solving method within a particular setting. This confirmation will provide, inturn, important insights for IT implementation theory and practice as well as into the project management discipline, through an ANT-based demonstration of the case and the problem-solving method.

\subsection{Expected Contributions}

Producing a workable method based on ANT would contribute to a widespread diffusion of numerous IT innovations by providing a specialized and highly efficient 
alternative to the traditional PMMs and IMTs currently used. Therefore, the ANT-based method for managing the initiation, production, and diffusion of innovations would strongly contribute to the advancement and specialization of the project management discipline.

Chiasson et al. (forthcoming) suggest that further work is required to determine the specific research epistemologies and their methodologies that are consistent with ARM. Demonstrating ANT's compatibility with ARM would promote further research work based on this research paradigm, producing an abundance of problem-solving methods for a number of practical settings.

\section{References}

Brown, D. 1997. Innovation Management Tools: A Review of Selected Methodologies, Luxembourg: European Commission.

Chiasson, M., Germonprez, M., and Mathiassen, L. Forthcoming. "Pluralist Action Research: A Review of the Information Systems Literature," Information Systems Journal.

Chiesa, V., Coughlan, P., and Voss, C. A. 1996. "Development of a Technical Innovation Audit," Journal of Product Innovation Management (13:2), pp. 105-136.

Cordella, A., and Shaikh, M. 2006. "From Epistemology to Ontology: Challenging the Constructed 'Truth' of ANT," working paper, Department of Information Systems, London School of Economics.

Hidalgo, A., and Albors J. 2008. "Innovation Management Techniques and Tools: A Review from Theory and Practice," R\&D Management (38:2), pp. 113-127.

Kautz, K., and. Nielsen, P. A. 2004. "Understanding the Implementation of Software Process Improvement Innovations in Software Organisations," Information Systems Journal (14:1), pp. 3-22.

Lanzara, G. 1999. "Designing Systems In-Action: Between Transient Constructs and Permanent Structures," keynote presentation, European Conference on Information Systems, Copenhagen, June 23-25.

Latour, B. 1987. Science in Action: How to Follow Scientists and Engineers through Society, Cambridge, MA: Harvard University Press.

Latour, B. 1999. "On Recalling ANT," in Actor Network Theory and After, J. Law and J. Hassard (eds.), Oxford, UK: Blackwell, pp. 15-25.

Latour, B. 2005. Reassembling the Social: An Introduction to Actor-Network-Theory, Oxford, UK: Oxford University Press.

Rogers, E, M. 1962. Diffusion of Innovation, New York: Free Press.

Slappendel, C. 1996. "Perspectives on Innovation in Organizations," Organization Studies (17), pp. 107-129.

Terré i Ohme, E. 2002. "Guide for Managing Innovation," Centre for Innovation and Business Development, Barcelona, Spain

Tuckman, B. 1965. "Developmental Sequence in Small Groups," Psychological Bulletin (63), pp. 384-399

\section{About the Authors}

Gerardo Zendejas is currently a Ph.D. student at the Department of Management Science, Management School, Lancaster University. Before joining Lancaster University, he held various 
managerial and consultant positions in both the health care and energy sectors. He also completed his Master of Business Administration with specialization in Management of Information Systems in the Haskayne School of Business, University of Calgary, in 2006, and a Bachelor's of Cybernetic Engineering and Computer Science at the La Salle University in 1995. His research examines and proposes methods to enhance focal actors' abilities to enroll and control the many social and technical components interacting during the initiation, production, and diffusion of innovations. By extension, his research focuses on understanding the reassembling and stabilizing of such components into the overall innovation process. His initial work has been qualitative in nature, using actor network theory extensively. Gerardo can be reached at gerardoz@, lancaster.ac.uk.

Mike Chiasson is currently an AIM (Advanced Institute of Management) Innovation Fellow and a Senior Lecturer at Lancaster University"s Management School, in the Department of Management Science. Before joining Lancaster University, he was an associate professor in the Haskayne School of Business, University of Calgary, and a postdoctoral fellow at the Institute for Health Promotion Research at the University of British Columbia. His research examines how social context affects IS development and implementation, using a range of social theories (actor network theory, structuration theory, critical social theory, ethnomethodology, communicative action, power knowledge, deconstruction, and institutional theory). In studying these questions, he has examined various development and implementation issues (privacy, user involvement, diffusion, outsourcing, cyber-crime, and system development conflict) within medical, legal, engineering, entrepreneurial, and governmental settings. Most of his work has been qualitative in nature, with a strong emphasis on participant observation. Mike can be reached at m.chiasson@1ancaster.ac.uk. 